\title{
Incidental genetic findings in randomized clinical trials: recommendations from the Genomics and Randomized Trials Network (GARNET)
}

\author{
Ebony B Bookman ${ }^{1, *}$, Corina Din-Lovinescu², Bradford B Worrall³, Teri A Manolio', Siiri N Bennett ${ }^{4}$, Cathy Laurie ${ }^{4}$, \\ Daniel B Mirel5 ${ }^{5}$, Kimberly F Doheny ${ }^{6}$, Garnet L Anderson? ${ }^{7}$ Kate Wehr ${ }^{8}$, Richard Weinshilboum ${ }^{9}$ and Donna T Chen ${ }^{10}$, \\ for GARNET
}

\begin{abstract}
Recommendations and guidance on how to handle the return of genetic results to patients have offered limited insight into how to approach incidental genetic findings in the context of clinical trials. This paper provides the Genomics and Randomized Trials Network (GARNET) recommendations on incidental genetic findings in the context of clinical trials, and discusses the ethical and practical issues considered in formulating our recommendations. There are arguments in support of as well as against returning incidental genetic findings in clinical trials. For instance, reporting incidental findings in clinical trials may improve the investigator-participant relationship and the satisfaction of participation, but it may also blur the line between clinical care and research. The issues of whether and how to return incidental genetic findings, including the costs of doing so, should be considered when developing clinical trial protocols. Once decided, plans related to sharing individual results from the aim(s) of the trial, as well as incidental findings, should be discussed explicitly in the consent form. Institutional Review Boards (IRBs) and other study-specific governing bodies should be part of the decision as to if, when, and how to return incidental findings, including when plans in this regard are being reconsidered.
\end{abstract}

\section{Introduction}

During clinical research, investigators may discover information with important health implications for a

*Correspondence: ebony.bookman@nih.gov

'National Human Genome Research Institute, 5635 Fishers Lane, Bethesda, MD 20892, USA

Full list of author information is available at the end of the article participant but that is not relevant to the initial aims of the study. Investigators and ethicists have been grappling with the question of how to address such incidental research findings for years. The ethical and practical considerations underlying this question are complex and often involve issues such as the researcher-participant relationship, the ethical practice of research, the cost and burden of follow-up, and participant expectations, among many other factors. The question of how to address incidental research findings becomes even more complicated in the context of clinical trials, in which participants may expect investigators to keep their medical best interests in mind, or researchers may feel obligated to report potentially important health findings to their participants. Clinical trials that involve genomic analyses add another level of complexity to the reporting of incidental findings because genomic studies can generate data on hundreds of thousands of genetic findings, most with unknown or evolving clinical significance. As the field of genomics continues to develop, there will be an increased need for shared experience and guidance on how to handle the discovery of incidental genetic research findings.

This paper discusses the process of developing the GARNET recommendations on incidental genetic findings in the context of clinical trials, and explores the ethical and practical issues considered in formulating our recommendations.

\section{GARNET}

GARNET comprises a series of genome-wide association studies (GWAS) of treatment responses in randomized clinical trials that aim to identify the genetic variants associated with response to treatments for conditions of clinical or public health significance [1]. The clinical trials that make up GARNET are the Women's Health Initiative (WHI) Hormone Therapy Trial, designed to identify genetic variations that alter the risk of coronary heart disease, stroke, venous thromboembolism and incident 
diabetes after exposure to hormone therapy; the SUCCESS A breast cancer trial, which aims to identify genetic variations that influence the efficacy or toxicity of chemotherapy for breast cancer; and the Vitamin Intervention for Stroke Prevention (VISP) trial, designed to identify genetic variants that influence the risk of recurrent stroke, myocardial infarction or death in response to vitamin therapy. GARNET utilizes existing clinical trial data and sample resources to identify genetic variants that influence an individual's response to treatment, to determine whether specific treatments are more or less effective in groups defined by genotype, and to develop and disseminate innovative methods for adding genomewide technologies to randomized clinical trials and interpreting the results in the context of a randomized treatment assignment. For a variety of historical reasons, consent forms for the clinical trials included in GARNET explicitly state that results will not be returned to participants. However, owing to investigator concerns about the possibility of encountering a potentially actionable genetic result, GARNET created an Incidental Findings Subcommittee to consider how to handle any such results.

After finding limited information on how to handle incidental genetic findings and individual research results when adding genome-wide analyses to clinical trials, the GARNET Incidental Findings Subcommittee set out to develop an approach as part of the goal of developing and disseminating methods for adding genome-wide technologies to randomized clinical trials (Box 1). The recommendations below are taken from $[1,2]$.

The GARNET recommendations were made after a review of the studies' consent forms and the existing literature regarding the return of individual research results and incidental findings in clinical trial and genetic research settings. From the outset, it was thought important to determine the kinds of incidental findings found when performing genome-wide genotyping, in order to provide information about this for researchers working on studies in which genomic analyses are prospectively or retrospectively incorporated into clinical trials. The precedent for this was set by the GENEVA consortium [3], which encourages research groups performing genome-wide association studies, such as GARNET, to think prospectively about how clinically relevant incidental findings will be addressed within their studies [4].

The Subcommittee also felt that IRBs and other studyspecific governing bodies should be actively involved when making decisions on when and how to report incidental genetic findings to individuals, since responsibility for overseeing study policies and procedures, investigator conduct, and participant safety is a shared responsibility. The Subcommittee felt that any return of results should be done in collaboration with a genetic medicine clinic because these clinics use Clinical Laboratory Improvement Amendments (CLIA)-certified laboratories, have experience with reporting genetic results to individuals, and have genetic counseling and referral resources at their disposal.

Of the total of 10,316 research samples that were genotyped in the three GARNET trials, incidental genetic findings were found in about $1 \%$ of samples after performing routine data cleaning and analysis. These included 132 large (>10 megabase pair (Mb)) autosomal chromosome anomalies in 109 samples, 1 male with Kleinfelter's syndrome (XXY), 7 females who had XXX or $\mathrm{XXX} / \mathrm{XX}$, and 16 females who were XX/XO. Some of the chromosomal anomalies detected in GARNET are similar to those found in the GENEVA GWAS consortium, where they were found to have an association with hematological cancers [5]. However, no GARNET findings were raised to an actionable level, given that reporting the large autosome anomalies detected has no clear clinical utility. The sex chromosome anomaly findings have possible treatment implications but were not raised to an actionable level as the anomalies may be acquired and, moreover, all participants with these anomalies were over reproductive age.

\section{Discussion}

In formulating our recommendations, the Subcommittee reviewed the ethical and practical considerations in support of reporting incidental genetic findings to clinical trial participants as well as the challenges inherent in doing so. Although no GARNET findings were raised to an actionable level, the issues we considered in making our recommendations apply to others facing similar deliberations.

\section{Considerations in support of reporting incidental genetic findings to clinical trial participants}

Although incidental findings in genetic studies have historically had a negative connotation due to misattributed parentage [6], recent studies show that incidental findings involving health-related information are not uniformly regarded as unwelcome by participants, particularly if the information can be used to prevent or treat a disease or disorder [7,8]. Indeed, two National Heart, Lung, and Blood Institute working groups recommend that individual genetic results should be offered to study participants if: (1) the genetic finding has important health implications for the participant, and the associated risks are established and substantial; (2) the finding is actionable; (3) the test is analytically valid; and (4) the study participant has opted to receive his or her genetic results $[9,10]$.

Ethical arguments supporting the disclosure of genetic research findings include: (1) the participant's interest in 


\section{Box 1. GARNET recommendations for incidental findings and potentially clinically relevant genetic results when} adding genomics to clinical trials

- Investigators are strongly encouraged to catalogue all identified incidental findings and/or potentially clinically relevant genetic abnormalities, results of discussions with IRBs, and actions taken, if any, so that all individuals involved in the study will have a sense of the scope of these issues.

- After identifying a potentially actionable incidental finding, investigators should discuss with their supervising IRBs how to handle the genetic research results in light of the study-specific consent forms.

- Future users of clinical trial data who identify findings that they consider to have clinical relevance should inform the primary study investigator of these findings, but must understand that all decisions related to notifying research participants of incidental or research genetic results reside with the primary study investigators and their IRBs.

- Investigators should use on-going national and international efforts that are coordinating the determination of clinical validity and utility/actionability of sequence and structural genetic variants to identify genetic findings that have 'established clinical relevance'.

- If research participants ask for their individual genetic results, and investigators and their IRBs have determined that no individual genetic results will be returned to participants, at a minimum the investigator should explain the study rules and try to determine the nature of the participant's interest. If appropriate, the investigators should refer individuals to a local genetic medicine clinic that offers genetic evaluation, diagnosis, assessment, genetic testing and interpretation, counseling and management, as well as referrals to appropriate resources for individuals with genetic disorders.

- Any return of genetic results to study participants should be done with the assistance of and in collaboration with a genetic medicine clinic that offers genetic evaluation, diagnosis, assessment, genetic testing and interpretation, counseling and management, as well as referrals to appropriate resources for individuals with genetic disorders.

- When preparing for further contact with participants in a research cohort (for example, informing participants of important findings that might affect their continued participation in a research study, as part of a re-consent process for the next phase of a longitudinal study, or even during a regularly scheduled periodic newsletter), as when designing new clinical trials with associated genetic studies, investigators are strongly encouraged to:

1. Discuss with their IRB how to present the possibility that there may be clinically relevant incidental and research findings, and outline circumstances and procedures for return of these results that respect the individual participant's preferences, local IRB requirements, and national recommendations;

2. Acknowledge in the consent forms and associated educational materials that this is an area of rapid change, and leave open the possibility that unforeseen circumstances may necessitate further discussion between investigators and participants; and

3. State in consent forms and associated educational materials that study participants may not be informed of important genetic results if investigators and their IRBs decide that no individual genetic results will be returned to participants.

information about themselves; (2) the benefit of receiving genetic findings, either because they can improve health or treatment, or because they can carry personal meaning; (3) an increase in trust in researchers from disclosure, ultimately leading to greater participation; (4) the principle of reciprocity - participants voluntarily agree to contribute to research, and should receive access to the knowledge gained from the research; and (5) returning results is a sign of respect that represents a core ethical commitment in research [11].

Beskow and Burke argue that different types of studies result in varying levels of obligation to return genetic results to participants [12]. For example, family-based studies or research in collaboration with rare disease organizations confer a stronger obligation for return of results, whereas secondary analysis of research data or biobanking studies may represent a weaker obligation [12]. Genetic research conducted in the context of clinical trials differs from many other genetic studies, and some of these contextual differences suggest a potentially stronger obligation for return of results.
The Department of Health and Human Services' Institutional Review Board Guidebook defines a clinical trial as 'a controlled study involving human subjects, designed to evaluate prospectively the safety and efficacy of new drugs or devices or of behavioral interventions' [13]. Participants often receive information about their clinical response, resulting in expectations that differ from observational studies. Thousands of people each year participate in clinical trials in the hope of furthering medical knowledge and obtaining needed medical care $[14,15]$. 'Therapeutic misconception', a widespread phenomenon, occurs when a research participant does not appreciate the distinction between the imperatives of clinical research and of medical treatment, and therefore inaccurately attributes therapeutic intent to research [16]. Because clinical trials are often performed at medical centers and by researchers who are also healthcare providers, participants may believe that they will receive healthcare benefits from their participation, regardless of the trial aims. Although the main goal of clinical research is to improve medical knowledge, many 
clinical trial participants believe the investigators will still provide the best standard of care [6]. A participant may also believe, when signing on to a clinical trial, that the investigators assume responsibility for their health, including that they will supply all relevant health information [17].

In clinical care, healthcare providers may find unexpected abnormalities that are unrelated to a patient's complaints but are essential to the patient's health [17]. The healthcare provider has a responsibility to disclose these findings to the patient, even though that was not the primary reason for the examination. Many feel that responsibility spills over to healthcare providers when they are in an investigator capacity [17]. Thus, some argue that researchers have an obligation to respond to incidental findings that arise during clinical research owing to the nature of the professional relationship they have with their participants and the general duty of beneficence [17]. When clinical trial designs are adapted based on results from interim analyses, such as in adaptive treatment-switching designs $[9,18,19]$, participants are given a greater chance of deriving medical benefit from their study participation and thus may also have increased expectations regarding disclosure of clinically relevant incidental finings, including incidental genetic findings when genetic analyses are included in the study.

Furthermore, some argue that the obligation to report individual results to participants increases when there is frequent interaction among researchers and participants, as in many clinical trials, because a more intense relationship creates a stronger ethical requirement for reciprocity [20]. Additionally, some believe that any increase in participants' vulnerability, uncompensated risks or burdens, and dependence on the researcher may increase the researcher's responsibility to provide ancillary care to participants [21]. As such, in clinical trials in which the researcher-participant relationship may be intense and the participant may be particularly vulnerable, the ethical duty to the participant, which may involve the return of incidental findings, is amplified.

\section{Challenges in reporting incidental genetic findings to clinical trial participants}

As in imaging studies, in which there may be incidental findings for a significant percentage of participants [22], participants in studies that involve genome-wide analyses may possess a vast number of variants that differ from reference sequences. Over 1,000 variants have been associated with a disease or trait and more than 650 variants have been associated with a drug response [23,24]. In contrast, hundreds of thousands of variants have no known trait or disease association. As more knowledge is obtained over time, the number of associations will increase, as each personal genome has more than
3 million single nucleotide polymorphisms and over 1,000 large ( $>500$ base pairs (bp)) copy number variants [25]. If clinical trials include whole exomes or wholegenome sequencing, the number of potential incidental genetic findings increases exponentially. When deciding which, if any, incidental genetic findings should be reported to individual study participants, it is important to consider the accuracy of the test results and the usefulness of the information to participants. These criteria are generally described in terms of analytic validity, clinical utility and clinical validity $[9,26]$.

Since the primary aim of research is to yield generalizable knowledge, some investigators feel that planning to disclose individual results such as incidental genetic findings further confuses the line between clinical care and research, and contributes to therapeutic misconception [27]. An appropriate disclosure policy requires that participants are educated beforehand about the range of possible findings and that consent forms truly inform the research participant of the risks and benefits of the proposed research, and explains all possible consequences from participating in the clinical trial. However, it is not feasible to define all the possible findings resulting from genomic analyses, as the number of possible incidental genetic findings is nearly infinite. It would also be impossible to describe the potential consequences of genomic analyses, because the implications of the majority of genetic variants found to date are not known. Finally, most genetic findings are probabilistic or pleiotropic, making them more complex for investigators to explain and for participants to comprehend [27].

Additionally, in genetic testing, there are often psychological effects on those receiving results. Participants receiving a positive test result may experience serious psychological burdens, while those receiving negative test results may experience a false sense of security and think that they will not develop the disease, not understanding that there is still a risk for disease roughly equal to the population risk [7]. Furthermore, even though the Genetic Information Nondiscrimination Act of 2008 (GINA) [28] is meant to decrease the possibility of discrimination in access to employment and health insurance associated with genetic findings, there remains a perceived threat of social stigmatization and discrimination in access to employment, health, disability and/or life insurance that has important implications for disclosing incidental genetic findings. These concerns also extend to family members of people receiving results [29].

Many investigators have stated that the practical difficulty of returning incidental findings is a major barrier to reporting back incidental findings to participants. The large amount of information garnered from incorporating genome-wide technology into clinical trials causes practical challenges for quality assurance, data handling 
and storage. For instance, the age and type of the specimen may affect test results, and cell lines made from primary tissue may exhibit chromosomal changes and mosaicism as an artifact of the transformation process. Sample handling and genotyping in most clinical trials is for research purposes only and is not performed under CLIA certification [30]. Current guidelines and regulations would require that investigators plan to have research results confirmed in a CLIA-certified laboratory to be appropriate for reporting to participants.

Finally, returning incidental genetic findings to individuals requires the investigator(s) who may be communicating the results to have appropriate genetic training and expertise [31,32]. Genetic risk for complex diseases can be particularly complicated and difficult to convey, leading to the common practice of deciding upfront not to return incidental genetic findings to participants and stating as much in the consent forms $[9,33]$. In a study looking at researcher perspectives on disclosure of incidental findings in genetic research, the surveyed investigators were concerned about the considerable time, energy and personnel necessary for re-contacting and reconsenting participants [34]. Disclosure of incidental findings can be resource intensive, and some argue that it would be unethical to use those resources for feedback when they could be used for research [35].

\section{Conclusion}

Although reporting incidental findings in clinical trials may improve the clinician-participant relationship and satisfaction of participation, it may blur the line between clinical care and research. As researchers, it is important to understand what kind of research information participants may want and to consider the scope and nature of our responsibility to communicate results from the aims of a study, as well as incidental genetic findings that may affect a person's health. These determinations are not straightforward and the environment is evolving rapidly. Investigators need to consider that there will be more and more opportunities for disclosure as the volume of genomic data in clinical trials research grows, our understanding of genetic contribution to health and illness expands, and practice norms surrounding giving genetic results to individual participants in research evolve. Investigators will also need to think carefully about when and how to present the possibility of discovering clinically relevant genomic findings that may not be the primary focus of the trial to study participants.

The issues of whether and how to return incidental genetic findings should be considered when determining which results from a trial will be reported. Once decided, plans related to sharing individual results from the aim(s) of the trial, as well as incidental findings, should be discussed explicitly in the consent form. IRBs and other study-specific governing bodies should be consulted when deciding if, when, and how to return incidental genetic findings, as they share responsibility with investigators for study policies and procedures, and participant safety. The GARNET experience and consensus approach to incidental findings may be of help in informing such decision-making in the future.

\section{Abbreviations}

CLIA, Clinical Laboratory Improvement Amendments; GARNET, Genomics and Randomized Trials Network; GINA, Genetic Information Nondiscrimination Act; GWAS, genome-wide association studies; IRB, Institutional Review Board; VISP, Vitamin Intervention for Stroke Prevention; WHI, Women's Health Initiative.

\section{Competing interests}

The authors declare that they have no competing interests.

\section{Acknowledgements}

The authors would like to thank the investigators within GARNET for their contribution to the GARNET Incidental Findings Subcommittee Recommendations as well as to this manuscript. We would also like to thank the NHGRI Return of Results consortium for their thoughtful comments and input. This work was supported in part by National Institutes of Health grants HG005137 (parent study SUCCESS A), HG005152 (parent study WHI), HG005160 (parent study VISP) and HG005157 (GARNET Coordinating Center).

\section{Author details}

'National Human Genome Research Institute, 5635 Fishers Lane, Bethesda, MD 20892, USA. ${ }^{2}$ Touro College of Osteopathic Medicine, 2090 Adam Clayton Powell Jr Blvd, New York, NY 10027, USA. ${ }^{3}$ Department of Neurology, Box 800394, University of Virginia School of Medicine, Charlottesville, VA 22908, USA. ${ }^{4}$ University of Washington, 46200 NE 74th Street, Bldg 29, Suite 310,

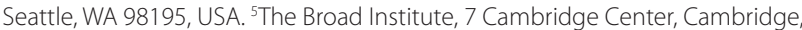
MA 02142, USA. ${ }^{\top}$ The Center for Inherited Disease Research, Johns Hopkins University, 333 Cassell Drive, Suite 2000, Baltimore, MD 21224, USA. ${ }^{7}$ Fred Hutchinson Cancer Research Center, 1100 Fairview Ave N, M3-A410, Seattle, WA 98109, USA. ${ }^{8}$ University of Washington, Box 359461, UW Tower 15th Floor, 4333 Brooklyn Ave NE, Seattle, WA 98195, USA. ${ }^{9}$ Mayo Medical School, Mayo Clinic, 200 First Street SW, Rochester, MN 55905, USA. ${ }^{\circ}$ Center for Biomedical Ethics and Humanities, Box 800758, University of Virginia School of Medicine, 101 Hospital Drive, Barringer 5380, Charlottesville, VA 22908, USA.

Published: 30 January 2013

\section{References}

1. GARNET [http://www.garnetstudy.org]

2. GARNET Statement on Incidental Findings and Potentially Clinically Relevant Genetic Results

[http://www.garnetstudy.org/Incidental_Findings_Table]

3. GENEVA Consortium [http://genevastudy.org]

4. Cornelis MC, Agrawal A, Cole JW, Hansel NN, Barnes KC, Beaty TH, Bennett SN, Bierut $L$, Boerwinkle E, Doheny KF, Feenstra B, Feingold E, Fornage M, Haiman CA, Harris EL, Hayes MG, Heit JA, Hu FB, Kang JH, Laurie CC, Ling H, Manolio TA, Marazita ML, Mathias RA, Mirel DB, Paschall J, Pasquale LR, Pugh EW, Rice JP, Udren J, et al:: The Gene, Environment Association Studies consortium (GENEVA): maximizing the knowledge obtained from GWAS by collaboration across studies of multiple conditions. Genet Epidemiol 2010, 34:364-372.

5. Laurie CC, Laurie CA, Rice K, Doheny KF, Zelnick LR, McHugh CP, Ling H, Hetrick KN, Pugh EW, Amos C, Wei Q, Wang LE, Lee JE, Barnes KC, Hansel NN, Mathias R, Daley D, Beaty TH, Scott AF, Ruczinski I, Scharpf RB, Bierut LJ, Hartz SM, Landi MT, Freedman ND, Goldin LR, Ginsburg D, Li J, Desch KC, Strom SS, Blot WJ, et al: Detectable clonal mosaicism from birth to old age and its relationship to cancer. Nat Genet 2012, 44:642-650.

6. Parker LS: The future of incidental findings: should they be viewed as benefits? J Law Med Ethics 2008, 36:341-351.

7. Shalowitz DI, Miller FG: The search for clarity in communicating research results to study participants. J Med Ethics 2008, 34:e17.

8. Kaufman D, Murphy J, Scott J, Hudson K: Subjects matter: a survey of public 
opinions about a large genetic cohort study. Genet Med 2008, 10:831-839.

9. Bookman EB, Langehorne AA, Eckfeldt JH, Glass KC, Jarvik GP, Klag M, Koski G, Motulsky A, Wilfond B, Manolio TA, Fabsitz RR, Luepker RV, NHLBI Working Group: Reporting genetic results in research studies: summary and recommendations of an NHLBI working group. Am J Med Genet A 2006, 140:1033-1040

10. Fabsitz RR, McGuire A, Sharp RR, Pandey M, Beskow LM, Biesecker LG, Bookman E, Burke W, Burchard EG, Church G, Clayton EW, Eckfeldt JH, Fernandez CV, Fisher R, Fullerton SM, Gabriel S, Gachupin F, James C, Jarvik GP, Kittles R, Leib JR, O'Donnell C, O'Rourke PP, Rodriguez LL, Schully SD, Shuldiner AR, Sze RKF, Thakuria JV, Wolf SM, Burke GL: Ethical and practical guidelines for reporting genetic research results to study participants: updated guidelines from a National Heart, Lung, and Blood Institute working group. Circ Cardiovasc Genet 2010, 3:574-580.

11. McGuire AL, Lupski JR: Personal genome research: what should the participant be told? Trends Genet 2010, 26:199-201.

12. Beskow $L M$, Burke $W$ : Offering individual genetic research results: context matters. Sci Trans/Med 2010, 2:38cm20.

13. Institutional Review Board Guidebook [http://www.hhs.gov/ohrp/archive/irb/irb_glossary.htm]

14. DeckW, Kosatsky T: Communicating their individual results to participant in an environmental exposure study: insights from clinical ethics. Environ Res 1999, 80:S223-S229.

15. Partridge AH, Wolff AC, Marcom PK, Kaufman PA, Zhang L, Gelman R, Moore C, Lake D, Fleming GF, Rugo HS, Atkins J, Sampson E, Collyar D, Winer EP: The impact of sharing results of a randomized breast cancer clinical trial with study participants. Breast Cancer Res Treat 2009, 115:123-129.

16. Lidz CW, Appelbaum PS: The therapeutic misconception: problems and solutions. Med Care 2002, 40:V55-63.

17. Miller FG, Mello MM, Joffe S: Incidental findings in human subjects research: what do investigators owe research participants? J Law Med Ethics 2008, 36:271-279.

18. Fernandez CV, Skedgel C, Weijer C: Considerations and costs of disclosing study findings to research participants. CMAJ 2004, 170:1417-1419.

19. Chow SC, Chang M: Adaptive design methods in clinical trials - a review. Orphanet J Rare Dis 2008, 3:11

20. Ravitsky $\mathrm{V}$, Wilfond $\mathrm{BS}$ : Disclosing individual genetic results to research participants. Am J Bioeth 2006, 6:8-17.

21. Belsky L, Richardson HS: Medical researchers' ancillary clinical care responsibilities. BMJ 2004, 328:1494-1496.

22. Devine AS, Jackson CS, Lyons L, Mason JD: Frequency of incidental findings on computed tomography of trauma patients. West J Emerg Med 2010 $11: 24-27$
23. Hindorff LA, Sethupathy P, Junkins HA, Ramos EM, Mehta JP, Collins FS, Manolio TA: Potential etiologic and functional implications of genomewide association loci for human diseases and traits. Proc Natl Acad Sci USA 2009, 106:9362-9367.

24. Johansson $A C$, Feuk L: Characterizing and interpreting genetic variation from personal genome sequencing. Methods Mol Biol 2012, 838:343-867.

25. Gonzaga-Jauregui C, Lupski JR, Gibbs RA: Human genome sequencing in health and disease. Annu Rev Med 2012, 63:35-61.

26. Burke W, Atkins D, Gwinn M, Guttmacher A, Haddow J, Lau J, Palomaki G, Press N, Richards CS, Wideroff L, Wiesner GL: Genetic test evaluation: information needs of clinicians, policy makers, and the public. Am J Epidemiol 2002, 156:311-318.

27. Bredenoord AL, Kroes HY, Cuppen E, Parker M, van Delden JJ: Disclosure of individual genetic data to research participants: the debate reconsidered. Trends Genet 2011, 27:41-47.

28. The Genetic Information Nondiscrimination Act of 2008 [http://www.eeoc.gov/laws/statutes/gina.cfm]

29. Hudson DL: Doctors' duty extends to family. Am Bar Assoc J 2004, eReport.

30. Clinical Laboratory Improvement Amendments [http://www.cms.hhs.gov/clia]

31. Smith K: Genetic testing of the general population: ethical and informatic concerns. Crit Rev Biomed Eng 2000, 28:557-561.

32. Mehlman MJ: Predictive genetic testing in urology: ethical and social issues. World J Urol 2004, 21:433-437.

33. Fullerton SM, Wolf WA, Brothers KB, Clayton EW, Crawford DC, Denny JC, Greenland P, Koenig BA, Leppig KA, Lindor NM, McCarty CA, McGuire AL, McPeek Hinz ER, Mirel DB, Ramos EM, Ritchie MD, Smith ME, Waudby CJ, Burke W, Jarvik GP: Return of individual research results from genome-wide association studies: experience of the Electronic Medical Records and Genomics (eMERGE) Network. Genet Med 2012, 14:424-431.

34. Meacham MC, Starks H, Burke W, Edwards K: Researcher perspectives on disclosure of incidental findings in genetic research. J Empir Res Hum Res Ethics 2010, 5:31-41.

35. Affleck P: Is it ethical to deny genetic research participants individualised results? J Med Ethics 2009, 35:209-213.

doi:10.1186/gm411

Cite this article as: Bookman EB, et al:: Incidental genetic findings in randomized clinical trials: recommendations from the Genomics and Randomized Trials Network (GARNET). Genome Medicine 2013, 5:7. 